\author{
Евгения Игоревна Суворова*, Светлана Анатольевна Шальнова, \\ Анна Васильевна Концевая, Александр Дмитриевич Деев, \\ Анна Владимировна Капустина, Юлия Андреевна Баланова \\ от имени участников исследования ЭССЕ-РФ
}

Национальный медицинский исследовательский центр профилактической медицины Россия, 101990, Москва, Петроверигский пер., 10

\begin{abstract}
Участники исследования ЭССЕ-РФ, соавторы статьи: Москва: Бойцов С.А., Чазов Е. И., Жернакова Ю.В., Гомыранова Н.В., Константинов В.В., Литинская О.А., Мамедов М.Н., Имаева А.Э., Муромцева Г.А., Евстифеева С.Е., Метельская В.А., Оганов Р.Г., Худяков М.Б., Ощепкова Е.В., Карпов Ю. А., Балахонова Т. В., Добровольский А. Б., Доценко А. Н., Еганян Р. А., Масенко В. П., Панченко Е. П., Постнов А. Ю., Пустеленин А. В., Рогоза А. Н., Титов В. Н. Санкт-Петербург: Шляхто Е.В., Конради А.О., Ротарь О.П., Баранова Е.И., Костарева А.А. Владивосток: Кулакова Н.В., Невзорова В.А., Шестакова Н.В., Мокшина М.В., Родионова Л.В., Владикавказ: Толпаров Г.В., Астахова 3. Т.; Вологда: Шабунова А.А., Калашников К.Н., Леонидова Г. В., Калачикова О.Н., Попов А.В., Ильин В.А., Россошанский А. И., Кондакова Н. А., Устинова К. А. Волгоград: Недогода С.В., Чумачек Е.В., Ледяева А.А., Воронеж: Черных Т.М., Фурменко Г.И., Минаков Э. В., Бондарцов Л.В., Бабенко Н.И., Азарин О.Г., Хвостикова А.Е. Иваново: Белова О.А., Романчук С.В., Назарова О.А., Шутемова Е.А., Корулина Л.В.; Кемерово: Барбараш О.Л., Артамонова Г.В., Индукаева Е.В., Максимов С.А., Скрипченко А.Е., Черкасс Н.В., Данильченко Я.В.; Красноярск: Гринштейн Ю.И., Петрова М.М., Данилова Л.К., Евсюков А.А., Топольская Н.В., Шабалин В.В., Аристов А.И., Руф Р.Р., Косинова А.А., Шматова Е.Н., Каскаева Д.С.; Оренбург: Либис Р.А., Исаева Е.Н., Басырова И.Р., Лопина Е.А., Кондратенко В.Ю., Сафонова Д.В.; Самара: Дупляков Д.В., Гудкова С.А., Черепанова Н.А. Томск: Трубачева И.А., Кавешников В.С., Карпов Р.С., Серебрякова В.Н.; Тюмень: Ефанов А.Ю., Медведева И.В., Сторожок М.А., Шалаев С.В.
\end{abstract}

\footnotetext{
Цель. Изучить ассоциации использования ресурсов системы здравоохранения и временной нетрудоспособности (ВН) с основными факторами риска (ФР) сердечно-сосудистых заболеваний (ССЗ) у лиц трудоспособного возраста по данным популяционного исследования ЭССЕ-РФ

Материал и методы. Анализ проведен на материале исследования ЭССЕ-РФ (13 регионов РФ). Использованы стандартные эпидемиологические методы обследования и критерии оценки. В анализ включены результаты опроса участников исследования об обращениях за медицинской помощью и ВН в течение предшествующих 12 мес. Рассчитывали среднее число амбулаторных обращений, госпитализаций (в том числе, длительность), вызовов скорой медицинской помощи (СМП) и ВН (количество дней и случаев) на 1 участника исследования и ассоциации с ФР ССЗ. Проводился сравнительный анализ распределений обращаемости за медицинской помощью и ВН, а также логистический регрессионный анализ для прогнозирования вероятности обращения к врачу, госпитализации, вызова СМП и оформления листа ВН в зависимости от наличия ФР ССЗ.

Результаты. Обследовано 21923 человека возраста 25-64 лет, мужчин 8373 (38\%), женщин 13550 (62\%). Количество мужчин, которые госпитализировались ( 1 раз и более), значимо возрастало в группе лиц, употреблявших табак $(1,22 ; p<0,05)$ и имевших ожирение $(1,32$; $p<0,05)$; вызывавших СМП также было значимо больше среди курящих $(1,41 ; p<0,05)$ и с повышенным уровнем глюкозы $(1,38 ; p<0,05)$; тогда как количество лиц, оформлявших лист ВН, статистически значимо увеличивалось только среди употреблявших табак $(1,29 ;$; $<0,05)$. Количество обращавшихся за медицинской помощью женщин, кроме госпитализации и оформлявших лист ВН, значимо увеличивалось только среди курящих. Частота обращений за медицинской помощью и наступления ВН в зависимости от наличия/отсутствия ФР, а также средняя длительность госпитализаций и ВН на 1 человека различалась незначительно как у мужчин, так и у женщин. Наличие ожирения у женщин - самый ресурсоемкий ФР, так же как курение у мужчин, и ассоциируется с увеличением вероятности использования всех типов медицинской помощи и оформления листов ВН. Курение и гипергликемия у женщин статистически значимо повышает вероятность использования большинства изучаемых ресурсов здравоохранения, тогда как у мужчин наличие гипергликемии повышает только вероятность вызова СМП $(1,38 ; p<0,05)$, а ожирение - госпитализации $(1,32 ; p<0,05)$. Повышенное артериальное давление статистически значимо влияет только на вызов СМП у обоих полов. Повышенный уровень общего холестерина у мужчин ассоциировался со снижением вероятности обращения за любым типом медицинской помощи.
} 
Заключение. Таким образом, в трудоспособном возрасте наблюдается статистически значимая ассоциация использования ресурсов системы здравоохранения с наличием ФР, которая варьирует в зависимости от типа медицинской помощи, пола и ФР.

Ключевые слова: факторы риска, расходование ресурсов системы здравоохранения, госпитализации, скорая медицинская помощь, обращения за медицинской помощью, врнеменная нетрудоспособность, трудоспособный возраст.

Для цитирования: Суворова Е.И., Шальнова С.А., Концевая А.В., Деев А.Д., Капустина А.В., Баланова Ю.А. от имени участников исследования ЭССЕ-РФ. Ассоциация использования ресурсов системы здравоохранения и временной нетрудоспособности с факторами риска хронических неинфекционных заболеваний в Российской Федерации по данным популяционного исследования. Рациональная фармакотерапия в кардиологии 2018;14(1):70-76. DOI: 10.20996/1819-6446-2018-14-1-70-76

\section{Association of risk factors for non-communicable diseases with health care resources utilization and temporary disability according to data of population study in Russian Federation}

Evgenia I. Suvorova*, Svetlana A. Shalnova, Anna V. Kontsevaya, Alexander D. Deev, Anna V. Kapustina, Yulia A. Balanova on behalf of the participants of the ESEE-RF study

National Medical Research Center for Preventive Medicine. Petroverigsky per. 10, Moscow, 101990 Russia

Aim. To analyze the associations of health care system resources utilization and temporary disability (TD) with the main risk factors (RF) for cardiovascular diseases (CVD) in working age population based on ESSE-RF study data.

Material and methods. The analysis was based on ESSE-RF study data (13 regions of the Russian Federation). Standard epidemiological survey methods and evaluation criteria were used. The analysis included results of a survey of the ESSE-RF study participants about the utilization of health services and TD during last 12 months. Average number of outpatient visits, hospitalizations (including duration of in-hospital treatment and number of cases), ambulance calls and TD (a number of days and cases) per one study participant and associations with RF for CVD were estimated. We conducted a comparative analysis of the utilization of health services and TD in association with cardiovascular RF, also logistic regression analysis was performed to predicting the likelihood of outpatient visits, hospitalizations, ambulance calls and TD.

Results. A total of 21,923 individuals in the 25-64 age group were included: men - 8,373 (38\%) and women - 13,550 (62\%). Number of men who was hospitalized significantly increased in group with tobacco consumption $(1,41 ; p<0,05)$ and obesity $(1,32 ; p<0,05)$, also number of participants who call to ambulance increased among smoking men $(1,41 ; p<0,05)$ and with hyperglycemia $(1,38 ; p<0,05)$, at the same time number of persons with cases of TD significantly increased just only among smokers $(1,29 ; p<0,05)$. At the same time, number of women who used health services (exclude in-hospital treatment) and who had a case of TD was significantly increased just only among smokers. The frequency of the utilization of health services and TD cases, the average number of days of hospitalizations and days of TD cases for 1 person depending on the presence/absence of RF had small difference in both men and women. Female obesity is the most resource-intensive RF, as well as male smoking, and was associated with an increase in the likelihood of using all types of medical care and TD cases. Smoking and hyperglycemia significantly increased the likelihood of utilization the most of health services in women, whereas in men hyperglycemia increased only the probability of ambulance calls $(1,38 ; p<0,05)$, and obesity - only hospitalizations $(1,32 ; p<0,05)$. Hypertension significantly affected only to ambulance calls in both men and women. Hypercholesterolemia was associated with decreased likelihood of the utilization health services in men.

Conclusion. So, there is a significant association of the health care utilization with the RF depending on the type of medical care, sex and RF.

Keywords: risk factors, health care resource utilization, hospitalizations, emergency, medical help seeking, working age.

For citation: Suvorova E.I., Shalnova S.A., Kontsevaya A.V., Deev A.D., Kapustina A.V., Balanova Y.A. on behalf of the participants of the ESEE-RF study. Association of risk factors for non-communicable diseases with health care resources utilization and temporary disability according to data of population study in Russian Federation. Rational Pharmacotherapy in Cardiology 2018;14(1):70-76. (In Russ). DOI: 10.20996/1819-6446-201814-1-70-76

*Corresponding author (Автор, ответственный за переписку): evgen-k@yandex.ru

Received / Поступила: 05.11.2017

Accepted / Принята в печать: 22.01.2018

Использование ресурсов системы здравоохранения и временной нетрудоспособности (ВН), как правило, изучается в клинических исследованиях как критерии эффективности лечения [1-2]. Вместе с тем эти параметры могут быть изучены в рамках эпидемиологических исследований с учетом влияния различных факторов риска (ФР) [3]. Известно, что сердечно-сосудистые заболевания (ССЗ) и их ФР связаны со значимыми социально-экономическими потерями, обусловленными расходованием ресурсов здравоохранения на оказание медицинской помощи пациентам и наступлением случаев ВН [5-7]. Эти дан- ные важны для оценки ущерба на популяционном уровне как неинфекционных заболеваний (что можно также изучать по данным официальной статистики), так и факторов риска (что данные статистики делать не позволяют).

По данным систематического обзора, объединившего результаты 15 исследований, было показано, что опрос позволяет получить достоверные данные об использовании ресурсов системы здравоохранения [4].

До настоящего времени подобного анализа об ассоциациях ФР ССЗ и ресурсов здравоохранения в Рос- 
сийской популяции трудоспособного возраста не проводилось.

Цель исследования: изучить ассоциации использования ресурсов системы здравоохранения и ВН с основными ФР ССЗ у лиц трудоспособного возраста по данным популяционного исследования ЭССЕ-РФ.

\section{Материал и методы}

Для анализа использованы данные многоцентрового наблюдательного исследования ЭССЕ-РФ, проведенного в 2012-1013 гг. в 13 регионах Российской Федерации. В рамках исследования были опрошены и обследованы представительные выборки населения в возрасте 25-64 лет из следующих регионов: Воронежская, Ивановская, Волгоградская, Оренбургская, Томская, Кемеровская, Тюменская области, города Самара, Санкт-Петербург, Владивосток, Вологда, Красноярск, Республика Северная Осетия-Алания. Методология исследования ЭССЕ-РФ, формирование представительных выборок и структура вопросника были подробно описаны ранее [8-10]. Исследование было одобрено независимыми этическими комитетами трех федеральных центров: Государственного научно-исследовательского центра профилактической медицины Минздрава России, Российского кардиологического научно-производственного комплекса Минздрава России, Федерального медицинского исследовательского центра им. В.А. Алмазова. У каждого участника было получено письменное информированное согласие на проведение обследования. Использованы стандартные эпидемиологические методы обследования и критерии оценки.

В рамках анализа участники исследования были поделены на группы в соответствии с наличием основных ФР: повышенное артериальное давление (АД), ожирение, повышенный уровень общего холестерина (ОХC), повышенный уровень глюкозы в крови, потребление табака (курение).

В качестве критериев деления на группы были приняты следующие положения:

- За повышенный уровень АД принимали значение систолического АД $\geqslant 140$ мм рт.ст. или диастолического АД $\geqslant 90$ мм рт.ст. [11], независимо от приема антигипертензивных препаратов.

- Ожирение определяли по величине индекса массы тела (ИМТ) $\geqslant 30 \mathrm{kг} / \mathrm{M}^{2}$;

- Повышенный уровень ОХС соответствовал концентрации этого показателя в сыворотке крови $\geqslant 5,0$ ммоль/л [10-11];

- Повышенный уровень глюкозы $\geqslant 7,0$ ммоль/л [10-11];

- По статусу курения участники поделены на лиц, никогда не потреблявших табак, и курящих, либо бро- сивших курить. Данные по потреблению табака получены с помощью стандартного вопросника, разработанного на основе адаптированных международных методик $[10,12]$.

Для оценки использования ресурсов системы здравоохранения анализировали результаты опроса участников исследования ЭССЕ-РФ О количестве обращений к врачу, госпитализациях, вызовах скорой медицинской помощи (СМП) в течение предшествующих 12 мес и ВН, а также длительность госпитализаций и ВН.

Статистический анализ. Статистическая обработка данных проводилась с использованием среды для статистических вычислений R 3.3.3. Определяли среднее количество обращений к врачу, случаев ВН (количество листов ВН), госпитализаций, вызовов СМП на 1 участника исследования, а также среднее количество дней ВН и госпитализаций.

Проводился сравнительный анализ распределений обращаемости за медицинской помощью и ВН в группах трудоспособного населения у лиц с изучаемыми ФР. В каждой группе рассчитывалась количество участников, 1 и более раз обратившихся к врачу, оформлявших лист ВН, госпитализированных и вызывавших СМП в течение 12 мес. В каждой группе проводилась процедура стандартизации прямым методом с использованием Европейского стандарта возрастной структуры населения.

Для прогнозирования вероятности обращения за медицинской помощью и наступления ВН в зависимости от наличия/отсутствия ФР С контролем на возраст был проведен логистический регрессионный анализ. В качестве объясняющих признаков были приняты следующие переменные: повышенное АД, ожирение, повышенный уровень ОХС, повышенный уровень глюкозы в крови, потребление табака. Зависимыми переменными выступали: факт обращения за медицинской помощью и наступление ВН. В результате проведения логистического регрессионного анализа были построены статистические модели для прогнозирования вероятности обращения к врачу, госпитализации, вызова СМП и оформления листа ВН, в которых были рассчитаны оценки регрессионных $\beta$-коэффициентов и вычислены отношения шансов (ОШ) для каждого изучаемого ФР для мужчин и для женщин.

\section{Результаты}

В анализ включены результаты обследования 21923 человек 25-64 лет, в том числе, 8373 (38\%) мужчин, и 13550 (62\%) женщин.

В табл. 1 представлены средние значения частоты обращений за медицинской помощью и частоты наступления случаев $\mathrm{BH}$, а также среднее количество 
Table 1. The frequency of visits to a doctor for medical care and temporary incapacity for work in a working-age population by risk factors (per person)

Таблица 1. Средние значения частоты обращений к врачу за медицинской помощью и ВН в популяции трудоспособного возраста по ФР (на 1 человека)

\begin{tabular}{|c|c|c|c|c|c|c|c|}
\hline \multirow[t]{2}{*}{ Мужчины } & & \multirow{2}{*}{$\begin{array}{c}\text { Обращение } \\
\text { к врачу }\end{array}$} & \multicolumn{2}{|c|}{ Госпитализация } & \multirow[t]{2}{*}{ Вызов СМП } & \multicolumn{2}{|c|}{ BH } \\
\hline & & & $\mathrm{n}$ & n дней & & $\mathrm{n}$ & $\mathrm{n}$ дней \\
\hline \multirow[t]{2}{*}{ Курение } & не курил & 2,2 & 1,3 & 17,0 & 1,4 & 1,3 & 16,2 \\
\hline & курил/курит & 2,3 & 1,3 & 17,8 & 1,5 & 1,3 & 16,3 \\
\hline \multirow[t]{2}{*}{ Уровень АД } & АД<140/90 & 2,2 & 1,3 & 17,9 & 1,4 & 1,3 & 16,4 \\
\hline & АД $\geqslant 140 / 90$ & 2,4 & 1,3 & 17,2 & 1,5 & 1,4 & 16,1 \\
\hline \multirow[t]{2}{*}{ ИМТ } & ИмТ $<30$ & 2,2 & 1,3 & 17,9 & 1,5 & 1,3 & 16,0 \\
\hline & ИMT $\geqslant 30$ & 2,5 & 1,3 & 16,9 & 1,4 & 1,4 & 16,8 \\
\hline \multirow[t]{2}{*}{ Уровень глюкозы в крови } & Глюкоза<7,0 & 2,2 & 1,3 & 17,4 & 1,5 & 1,3 & 16,3 \\
\hline & Глюкоза $\geqslant 7,0$ & 3,3 & 1,2 & 17,7 & 1,4 & 1,4 & 16,4 \\
\hline \multirow[t]{2}{*}{ Общий холестерин } & $x C<5,0$ & 2,3 & 1,3 & 18,3 & 1,4 & 1,4 & 16,9 \\
\hline & $X C \geqslant 5,0$ & 2,3 & 1,2 & 16,9 & 1,5 & 1,3 & 15,8 \\
\hline \multicolumn{2}{|l|}{ Все мужчины } & 2,3 & 1,3 & 17,5 & 1,5 & 1,3 & 16,3 \\
\hline \multirow[t]{2}{*}{ Женщины } & & \multirow{2}{*}{$\begin{array}{c}\text { Обращение } \\
\text { к врачу }\end{array}$} & \multicolumn{2}{|c|}{ Госпитализация } & \multirow[t]{2}{*}{ Вызов СМП } & \multicolumn{2}{|c|}{$\mathrm{BH}$} \\
\hline & & & $\mathrm{n}$ & $\mathrm{n}$ дней & & $\mathrm{n}$ & n дней \\
\hline \multirow[t]{2}{*}{ Курение } & не курил & 2,8 & 1,2 & 17,4 & 1,9 & 1,4 & 17,5 \\
\hline & курил/курит & 2,8 & 1,2 & 15,9 & 1,8 & 1,5 & 17,8 \\
\hline \multirow[t]{2}{*}{ Уровень АД } & АД<140/90 & 2,7 & 1,2 & 16,7 & 1,8 & 1,4 & 17,7 \\
\hline & АД $\geqslant 140 / 90$ & 3,1 & 1,2 & 17,5 & 2,1 & 1,4 & 17,4 \\
\hline \multirow[t]{2}{*}{ ИMT } & ИМТ $<30$ & 2,6 & 1,2 & 16,6 & 1,7 & 1,4 & 17,3 \\
\hline & ИMT $\geqslant 30$ & 3,1 & 1,3 & 17,8 & 2,2 & 1,4 & 18,1 \\
\hline \multirow[t]{2}{*}{ Уровень глюкозы в крови } & Глюкоза $>7,0$ & 2,7 & 1,2 & 17,1 & 1,8 & 1,4 & 17,7 \\
\hline & Глюкоза $\geqslant 7,0$ & 4,6 & 1,3 & 17,4 & 2,5 & 1,6 & 17,4 \\
\hline \multirow[t]{2}{*}{ Общий холестерин } & $X C<5,0$ & 2,6 & 1,2 & 15,9 & 1,8 & 1,4 & 15,9 \\
\hline & $X C \geqslant 5,0$ & 3 & 1,2 & 17,7 & 2 & 1,4 & 18,7 \\
\hline \multicolumn{2}{|l|}{ Все женщины } & 2,8 & 1,2 & 17,1 & 1,9 & 1,4 & 17,6 \\
\hline \multicolumn{2}{|l|}{ Bce } & 2,6 & 1,2 & 17,2 & 1,8 & 1,4 & 17,1 \\
\hline
\end{tabular}

ИМТ - индекс массы тела, АД - артериальное давление, ХС - холестерин, СМП - скорая медицинская помощь, ВН - временная нетрудоспособность

дней госпитализации и ВН на 1 человека для мужчин и для женщин 25-64 лет. У мужчин среднее число обращений к врачу было несколько меньше, чем у женщин, и составило 2,3 раза в год на одного человека против 2,8, мужчины госпитализировались в среднем немного чаще женщин ( 1,3 против 1,2 раза в год, соответственно), хотя число вызовов СМП в группах мужчин было на 0,4 раза меньше женщин (1,5 и 1,9, соответственно). Вместе с тем, количество случаев ВН 1,3 (1,3-1,4) у мужчин было зарегистрировано меньше, чем у женщин - 1,4 (1,4-1,5), пребывание на больничном листе у мужчин было также менее продолжительным, и составило 16,3 (15,8-16,9) дней по сравнению с 17,6 (15,9-18,7) дней у женщин.

Различия между группами в зависимости от наличия и отсутствия ФР наблюдались как у мужчин, так и у женщин, и варьировали в зависимости от ФР и вида медицинской помощи.
При анализе стандартизованных показателей обращаемости за медицинской помощью (табл. 2), оказалось, что у мужчин три ФР (курение, ожирение и гипергликемия) ассоциированы со статистически значимым увеличением использования ресурсов системы здравоохранения, в то время как у женщин только один (курение). Так, среди курящих мужчин экстренная медицинская помощь, стационарное лечение и оформление листа ВН потребовались большему числу лиц, чем в группе лиц, никогда не употреблявших табак. Похожая ситуация наблюдалась и у женщин, где среди курящих значимо возрастало количество тех, кто обращался к врачу $(62,9$ против $57,4)$, вызывал СМП $(13,0)$ и оформлял лист $\mathrm{BH}(23,4)$ хотя бы один раз за предшествующие 12 мес. В группе мужчин с повышенным уровнем глюкозы значимо возрастало число лиц, которые вызывали СМП и госпитализировались, а в группе с ожирением возрастало 
Table 2. The uptake of medical care and temporary disability in the working age population on risk factors (for 100 people) Таблица 2. Обращаемость за медицинской помощью и ВН в популяции трудоспособного возраста по ФР (на 100 человек) 1

\begin{tabular}{|c|c|c|c|c|c|c|c|c|c|}
\hline \multirow{2}{*}{ Мужчины } & & \multicolumn{2}{|c|}{ Обращение к врачу } & \multicolumn{2}{|c|}{ Госпитализация } & \multicolumn{2}{|c|}{ Вызов СМП } & \multicolumn{2}{|c|}{$\mathrm{BH}$} \\
\hline & & $\mathrm{n}$ & 95\% ди & $\mathrm{n}$ & $95 \%$ ди & $\mathrm{n}$ & $95 \%$ ди & $\mathrm{n}$ & $95 \%$ ди \\
\hline Курение & $\begin{array}{l}\text { не курил } \\
\text { курил/курит }\end{array}$ & $\begin{array}{l}43,5 \\
46,5\end{array}$ & $\begin{array}{l}41,1-46,1 \\
44,7-48,3\end{array}$ & $\begin{array}{l}7,2 \\
9,1^{*}\end{array}$ & $\begin{array}{l}6,2-8,2 \\
8,3-9,9\end{array}$ & $\begin{array}{l}5,7 \\
8,3^{*}\end{array}$ & $\begin{array}{l}4,9-6,7 \\
7,5-9,1\end{array}$ & $\begin{array}{l}16,3 \\
19,9^{*}\end{array}$ & $\begin{array}{l}14,8-17,9 \\
18,7-21,2\end{array}$ \\
\hline Уровень АД & $\begin{array}{l}A Z<140 / 90 \\
A Z \geqslant 140 / 90\end{array}$ & $\begin{array}{l}45,1 \\
45,9 \\
\end{array}$ & $\begin{array}{l}43,2-47,1 \\
43,4-48,5 \\
\end{array}$ & $\begin{array}{l}8,3 \\
8,8 \\
\end{array}$ & $\begin{array}{l}7,4-9,1 \\
7,7-9,9 \\
\end{array}$ & $\begin{array}{l}6,8 \\
8,6 \\
\end{array}$ & $\begin{array}{l}6,0-7,6 \\
7,5-9,7 \\
\end{array}$ & $\begin{array}{l}19,0 \\
18,7 \\
\end{array}$ & $\begin{array}{l}17,7-20,3 \\
17,0-20,4 \\
\end{array}$ \\
\hline ИМТ & $\begin{array}{l}\text { ИМТ }<30 \\
\text { ИМТ } \geqslant 30\end{array}$ & $\begin{array}{l}44,7 \\
47,8\end{array}$ & $\begin{array}{l}43,0-46,4 \\
44,7-51,0\end{array}$ & $\begin{array}{l}7,8 \\
9,9 *\end{array}$ & $\begin{array}{l}7,1-8,5 \\
8,7-11,4\end{array}$ & $\begin{array}{l}7,0 \\
8,4\end{array}$ & $\begin{array}{l}6,4-7,7 \\
7,2-9,8\end{array}$ & $\begin{array}{l}18,5 \\
19,3\end{array}$ & $\begin{array}{l}17,5-19,7 \\
17,3-21,4\end{array}$ \\
\hline Уровень глюкозы в крови & $\begin{array}{l}\text { Глюкоза }<7,0 \\
\text { Глюкоза }>7,0 \\
\end{array}$ & $\begin{array}{l}45,4 \\
49,1 \\
\end{array}$ & $\begin{array}{l}43,9-47,0 \\
41,6-58,1 \\
\end{array}$ & $\begin{array}{c}8,3 \\
13,7^{*} \\
\end{array}$ & $\begin{array}{l}7,7-9,0 \\
9,7-19,4 \\
\end{array}$ & $\begin{array}{c}7,1 \\
11,5^{*} \\
\end{array}$ & $\begin{array}{l}6,6-7,8 \\
8,0-16,6 \\
\end{array}$ & $\begin{array}{l}18,8 \\
19,1 \\
\end{array}$ & $\begin{array}{l}17,8-19,8 \\
14,3-25,5 \\
\end{array}$ \\
\hline Общий холестерин & $\begin{array}{l}X C<5,0 \\
X C \geqslant 5,0\end{array}$ & $\begin{array}{l}47,1 \\
45,2 \\
\end{array}$ & $\begin{array}{l}44,7-49,6 \\
43,3-47,3 \\
\end{array}$ & $\begin{array}{l}9,4 \\
8,2 \\
\end{array}$ & $\begin{array}{c}8,3-10,5 \\
7,4-9,1 \\
\end{array}$ & $\begin{array}{l}8,0 \\
7,0 \\
\end{array}$ & $\begin{array}{l}7,1-9,1 \\
6,3-7,8 \\
\end{array}$ & $\begin{array}{l}18,7 \\
19,2 \\
\end{array}$ & $\begin{array}{l}17,2-20,3 \\
17,9-20,5 \\
\end{array}$ \\
\hline \multirow[t]{2}{*}{ Женщины } & & \multicolumn{2}{|c|}{ Обращение к врачу } & \multicolumn{2}{|c|}{ Госпитализация } & \multicolumn{2}{|c|}{ Вызов СМП } & \multicolumn{2}{|c|}{$\mathrm{BH}$} \\
\hline & & $\mathrm{n}$ & $95 \%$ ди & $n$ & $95 \%$ ди & $\mathrm{n}$ & $95 \%$ ди & $\mathrm{n}$ & 95\% ди \\
\hline Курение & $\begin{array}{l}\text { не курил } \\
\text { курил/курит }\end{array}$ & $\begin{array}{l}57,4 \\
62,9^{*}\end{array}$ & $\begin{array}{l}55,8-59,0 \\
60,1-65,7\end{array}$ & $\begin{array}{c}9,4 \\
10,5\end{array}$ & $\begin{array}{l}8,8-10,0 \\
9,4-11,7\end{array}$ & $\begin{array}{c}9,9 \\
13,0^{*}\end{array}$ & $\begin{array}{c}9,3-10,5 \\
11,7-14,3\end{array}$ & $\begin{array}{l}20,6 \\
23,4^{*}\end{array}$ & $\begin{array}{l}19,6-21,6 \\
21,7-25,2\end{array}$ \\
\hline Уровень АД & $\begin{array}{l}\text { АД }<140 / 90 \\
\text { АД } \geqslant 140 / 90\end{array}$ & $\begin{array}{l}58,1 \\
59,6\end{array}$ & $\begin{array}{l}56,5-59,7 \\
56,0-63,4\end{array}$ & $\begin{array}{c}9,2 \\
10,5\end{array}$ & $\begin{array}{l}8,6-9,9 \\
9,0-12,3\end{array}$ & $\begin{array}{c}9,8 \\
11,8\end{array}$ & $\begin{array}{c}9,2-10,5 \\
10,4-13,4\end{array}$ & $\begin{array}{l}21,1 \\
20,6\end{array}$ & $\begin{array}{l}20,2-22,2 \\
18,6-23,0\end{array}$ \\
\hline ИМТ & $\begin{array}{l}\text { ИMT }<30 \\
\text { ИMT } \geqslant 30\end{array}$ & $\begin{array}{l}57,7 \\
60,9 \\
\end{array}$ & $\begin{array}{l}56,1-59,4 \\
57,8-64,3 \\
\end{array}$ & $\begin{array}{l}9,1 \\
9,5 \\
\end{array}$ & $\begin{array}{l}8,5-9,7 \\
8,4-10,7 \\
\end{array}$ & $\begin{array}{c}9,8 \\
11,6 \\
\end{array}$ & $\begin{array}{c}9,1-10,4 \\
10,4-13,0 \\
\end{array}$ & $\begin{array}{l}20,7 \\
22,6 \\
\end{array}$ & $\begin{array}{l}19,7-21,7 \\
20,6-24,8 \\
\end{array}$ \\
\hline Уровень глюкозы в крови & $\begin{array}{l}\text { Глюкоза }<7,0 \\
\text { Глюкоза } 7,0\end{array}$ & $\begin{array}{l}58,7 \\
62,0\end{array}$ & $\begin{array}{l}57,3-60,1 \\
51,9-75,1 \\
\end{array}$ & $\begin{array}{c}9,5 \\
11,4 \\
\end{array}$ & $\begin{array}{l}9,0-10,1 \\
7,6-18,8 \\
\end{array}$ & $\begin{array}{l}10,5 \\
13,6 \\
\end{array}$ & $\begin{array}{l}9,9-11,1 \\
9,5-21,3 \\
\end{array}$ & $\begin{array}{l}21,2 \\
22,4 \\
\end{array}$ & $\begin{array}{l}20,3-22,1 \\
16,3-32,0\end{array}$ \\
\hline Общий холестерин & $\begin{array}{l}X C<5,0 \\
X C \geqslant 5,0\end{array}$ & $\begin{array}{l}59,3 \\
59,5\end{array}$ & $\begin{array}{l}57,1-61,5 \\
57,6-61,6\end{array}$ & $\begin{array}{c}9,3 \\
10,2\end{array}$ & $\begin{array}{l}8,5-10,3 \\
9,4-11,1\end{array}$ & $\begin{array}{l}10,6 \\
10,8\end{array}$ & $\begin{array}{c}9,7-11,6 \\
10,0-11,6\end{array}$ & $\begin{array}{l}20,9 \\
21,6\end{array}$ & $\begin{array}{l}19,6-22,2 \\
20,3-22,9\end{array}$ \\
\hline $\begin{array}{l}\text { 'стандартизованная по возрас } \\
\text { *p<0,05 по сравнению с не и } \\
\text { ИМТ - индекс массы тела, AD }\end{array}$ & $\begin{array}{l}\text { Ту } \\
\text { меющими ФР } \\
\text { - артериальнс }\end{array}$ & & & & & & 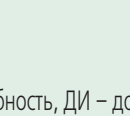 & & \\
\hline
\end{tabular}

только количество тех, кому потребовалось лечение в условиях стационара. По остальным показателям статистически значимых различий не было выявлено.

При проведении логистического регрессионного анализа показано, что вероятность обращения к врачу у мужчин статистически значимо прогнозирует потребление табака $(1,12 ; p<0,05)$ и повышенный уровень OXC $(0,89 ; p<0,05)$, в то время как у женщин это - потребление табака $(1,26 ; p<0,05)$, ожирение $(1,12 ; p<0,05)$ и высокий уровень глюкозы в крови $(1,65 ; p<0,05)$ (табл. 3). Статистически значимо чаще госпитализируются курящие мужчины $(1,22 ; p<0,05)$, а также мужчины, имеющие ожирение $(1,32 ; p<0,05)$ и высокий уровень OXC $(0,85 ; p<0,05)$, у женщин факторами, статистически значимо ассоциированными с госпитализациями, оказались ожирение $(1,16$; $p<0,05)$ и высокий уровень глюкозы $(1,67 ; p<0,05)$. Все изучаемые ФР статистически значимо прогнозируют вероятность вызова СМП (кроме ожирения у мужчин и повышенного уровня ОХС у женщин). Вероятность наступления случаев ВН у мужчин значимо увеличивает только потребление табака (1,29; $p<0,05)$, тогда как у женщин - потребление табака $(1,17 ; p<0,05)$ и ожирение $(1,14 ; p<0,05)$.

\section{Обсуждение}

Анализ использования ресурсов системы здравоохранения и ВН на популяционном уровне в рамках исследования ЭССЕ-РФ позволил исследовать ассоциации обращений за медицинской помощью и ВН в течение 1 года с наличием основных ФР ССЗ у лиц трудоспособного возраста. Ряд факторов риска ассоциирован со статистически значимым увеличением обращаемости за медицинской помощью, этими факторами оказались курение, ожирение, гипергликемия, в меньшей степени - повышенное АД. Сходные результаты получены в крупном популяционном исследовании в США, где было показано, что наличие ФР ассоциировалось с большей вероятностью обращения за медицинской помощью и потреблением ресурсов системы здравоохранения [13-14].

Курение - один из самых опасных ФР, в списке заболеваний, вызываемых курением - СС3, рак, респираторные болезни и другие заболевания. Поэтому не 
Table 3. Results of logistic regression analysis of the probability of treatment for medical help and temporary disablement Таблица 3. Результаты логистического регрессионного анализа вероятности обращения за медицинской помощью и $\mathrm{BH}$

\begin{tabular}{|c|c|c|c|c|c|c|c|c|}
\hline \multirow[t]{2}{*}{ Мужчины } & \multicolumn{2}{|c|}{ Обращение к врачу } & \multicolumn{2}{|c|}{ Госпитализация } & \multicolumn{2}{|c|}{ Вызов СМП } & \multicolumn{2}{|c|}{$\mathrm{BH}$} \\
\hline & ОШ & $95 \%$ ди & Ош & $95 \%$ ди & ОШ & $95 \%$ ди & Ош & $95 \%$ ди \\
\hline Курение & $1,12^{*}$ & $1,02-1,24$ & $1,22^{*}$ & $1,03-1,45$ & $1,41^{*}$ & $1,17-1,71$ & $1,29 *$ & $1,14-1,46$ \\
\hline Уровень АД & 1,03 & $0,93-1,13$ & 0,96 & $0,81-1,14$ & $1,21^{*}$ & $1,01-1,46$ & 0,96 & $0,85-1,09$ \\
\hline ИMT & 1,10 & $0,99-1,22$ & $1,32^{*}$ & $1,11-1,57$ & 1,16 & $0,96-1,40$ & 1,07 & $0,93-1,22$ \\
\hline Уровень глюкозы в крови & 1,14 & $0,94-1,39$ & 1,22 & $0,91-1,61$ & $1,38 *$ & $1,02-1,84$ & 0,97 & $0,76-1,24$ \\
\hline Общий холестерин & $0,89 *$ & $0,81-0,98$ & $0,85^{*}$ & $0,72-1,00$ & $0,84^{*}$ & $0,70-0,99$ & 1,05 & $0,93-1,18$ \\
\hline Включено в анализ & & 7823 & & 7811 & & 7813 & & \\
\hline \multirow[t]{2}{*}{ Женщины } & \multicolumn{2}{|c|}{ Обращение к врачу } & \multicolumn{2}{|c|}{ Госпитализация } & \multicolumn{2}{|c|}{ Вызов СМП } & \multicolumn{2}{|c|}{$\mathrm{BH}$} \\
\hline & ОШ & $95 \%$ ди & Ош & $95 \%$ ди & ОШ & 95\% ди & Ош & 95\% ди \\
\hline Курение & $1,26 *$ & $1,15-1,37$ & 1,13 & $0,99-1,30$ & $1,36 *$ & $1,20-1,55$ & $1,17^{*}$ & $1,06-1,30$ \\
\hline Уровень АД & 1,08 & $0,99-1,17$ & 1,13 & $1,00-1,29$ & $1,21^{*}$ & $1,08-1,37$ & 1,00 & $0,90-1,11$ \\
\hline ИMT & $1,12^{*}$ & $1,03-1,22$ & $1,16^{*}$ & $1,03-1,32$ & $1,26 *$ & $1,12-1,41$ & $1,14^{*}$ & $1,04-1,26$ \\
\hline Уровень глюкозы в крови & $1,65^{*}$ & $1,38-1,96$ & $1,67^{*}$ & $1,36-2,04$ & $1,39 *$ & $1,14-1,69$ & 1,01 & $0,82-1,22$ \\
\hline Общий холестерин & 0,97 & $0,89-1,04$ & 1,04 & $0,91-1,18$ & 0,96 & $0,85-1,08$ & 1,08 & $0,98-1,19$ \\
\hline Включено в анализ & \multicolumn{2}{|r|}{13029} & \multicolumn{2}{|c|}{12993} & \multicolumn{2}{|c|}{13001} & \multicolumn{2}{|c|}{12592} \\
\hline
\end{tabular}

удивительно, что в нашем исследовании показано, что потребление табака статистически значимо увеличивает вероятность обращения за всеми видами медицинской помощи и наступления ВН, кроме вероятности госпитализации женщин. Об этом же сообщают Wacker с соавт. [6] и Vals с соавт. [15], которые продемонстрировали статистически значимую связь потребления табака с использованием ресурсов системы здравоохранения в популяции трудоспособного возраста Германии и Эстонии.

Повышенный уровень АД неожиданно мало ассоциирован со статистически значимым увеличением обращений за медицинской помощью, так значимо возрастает только вероятность вызова СМП независимо от пола. В исследовании, проведенном Natarajan S., Nietert P.J. продемонстрировано, что среди жителей США с повышенным АД также статистически значимо увеличивалась вероятность обращения в отделение скорой помощи [14].

Значимость ожирения как ФР существенно возросла в РФ [16]. Ожирение среди женщин - один из самых ресурсоемких ФР, вносящий значимый вклад в повышенное использование всех видов медицинской помощи, и также увеличивающий вероятность наступления ВН, в то время как мужчины с ожирением чаще госпитализируются. Распространение ожирения способствует увеличению нагрузки на систему здравоохранения, что подтверждается результатами исследований, проведенных в США, Эстонии и Австралии [15, 17-18].
Исследователи из США, изучавшие влияние ФР на потребление ресурсов здравоохранения, отмечают, что наибольшая вероятность обращения за медицинской помощью наблюдается среди лиц с высоким уровнем глюкозы в крови (>125 мг/дл или >7,0 ммоль/л) $[7,14]$. В нашем исследовании были получены схожие данные у женщин, тогда как у мужчин эти показатели с высокой степенью вероятности ассоциировались только с госпитализацией.

Мы получили обратную зависимость между уровнем OXC и использованием ресурсов здравоохранения у мужчин. Существует ряд зарубежных исследований, результаты которых показывают статистически значимую связь низкого уровня ОХС с увеличением смертности от ССЗ [19-20]. В ходе анализа было выявлено, что повышенный уровень ОХС ассоциирован со снижением вероятности обращения за медицинской помощью у мужчин, что, возможно, косвенно связано с увеличенным показателем смертности среди лиц с пониженным ОХС [19-20].

Таким образом, полученные результаты демонстрируют статистически значимые ассоциации ФР с расходованием ресурсов здравоохранения, несколько различные у мужчин и женщин.

\section{Заключение}

Впервые на популяционном уровне изучено использование ресурсов системы здравоохранения у лиц трудоспособного возраста, а также зависимость различных вариантов обращения за медицинской по- 
мощью и ВН с основными ФР ССЗ. Полученные результаты будут использованы для оценки экономического бремени ФР ССЗ. Увеличение инвестиций в профилактику, прежде всего популяционную, позволит достичь снижения распространенности ФР и затрат на лечение в будущем.

\section{References / Литература}

1. Cook J., Drummond M., Heyse J.F. Economic endpoints in clinical trials. Statistical Methods in Medical Research. 2004;13:157-76. doi: 10.1093/epirev/24.1.80.

2. Ramsey S, Willke R, Briggs A, Good research practices for cost-effectiveness analysis alongside clinical trials: the ISPOR RCT-CEA Task Force report. Value Health. 2005;8(5):521-33. doi: 10.1111/j.1524-4733.2005.00045.x.

3. Boccolini C.S., de Souza Junior P.R. Inequities in Healthcare utilization: results of the Brazilian National Health Survey, 2013. International Journal for Equity in Health. 2016;15:150. doi: 10.1186/s12939016-0444-3.

4. Leggett L.E., Khadaroo R.G., Holroyd-Leduc J. et al. Measuring Resource Utilization: A Systematic Review of Validated Self-Reported Questionnaires. Medicine 2016; 95(10):e2759.

doi: 10.1097/MD.0000000000002759.

5. Oganov R.G., Kontsevaya A.V., Kalinina A.M. State Economic Burden of Cardiovascular Diseases in the Russian Federation. Cardiovascular Therapy and Prevention. 2011;10(4):4-9. (In Russ.) [Оганов Р.Г., Концевая А.В., Калинина А.М. Экономический ущерб от сердечно-сосудистых заболеваний в Российской Федерации. Кардиоваскулярная Терапия и Профилактика. 2011;10(4):4-9].

6. Wacker M., Holle R., Heinrich J. et al. The association of smoking status with healthcare utilisation, productivity loss and resulting costs: results from the population-based KORA F4 study. BMC Health Services Research. 2013;13:278. doi: 10.1186/1472-6963-13-278.

7. Boudreau D.M., Malone D.C., Raebel M.A. et al. Health care utilization and costs by metabolic syndrome risk factors. Metabolic syndrome and related disorders. 2009;7(4):305-14. doi: 10.1089/met.2008.0070.

8. Boitsov S.A., Chazov E.I., Shlyakhto E.V., Shalnova S.A. et al. Epidemiology of cardiovascular diseases in different regions of Russia (ESSE-RF). The rationale for and design of the study. Profilakticheskaya Meditsina. 2013;16(6):25-34. (In Russ.) [Бойцов С.А., Чазов Е. И., Шляхто Е.В., Шальнова С.А. и др. Эпидемиология сердечно-сосудистых заболеваний в различных регионах России (ЭССЕРФ). Обоснование и дизайн исследования. Профилактическая Медицина. 2013;16(6):25-34].

9. Boytsov S. A., Balanova Yu. A., Shalnova S. A. et al. Arterial hypertension among individuals of 25-64 years old: prevalence, awareness, treatment and control. By the data from ECCD. Cardiovascular Therapy and Prevention. 2014;13(4):4-14. (In Russ.) [Бойцов С.А., Баланова Ю.А., Шальнова С.А. и др. Артериальная гипертония среди лиц 25-64 лет: распространенность, осведомленность, лечение и контроль. По материалам исследования ЭССЕ. Кардиоваскулярная Терапия И Профилактика. 2014;13(4):4-14.] doi: 10.15829/1728-8800-2014-4-4-14.

10. Muromtseva G.A., Kontsevaya A.V., Konstntinov V.V., et al. The prevalence of non-infectious diseases risk factors in russian population in 2012-2013 years. The results of ECVD-RF. Cardiovascular Therapy and Prevention. 2014;13(6):4-11. (In Russ.) [Муромцева Г.А., Концевая А.В., Константинов В.В. и др. Распространенность факторов риска неинфекционных заболеваний в российской популяции в 2012-2013гг. Результаты исследования ЭССЕ-РФ. Кардиоваскулярная Терапия и Профилактика. 2014;13(6):4-11] doi: 10.15829/1728-8800-2014-6-4-11.

\section{About the Authors:}

Evgenia I. Suvorova - Junior Researcher, Laboratory of Economic Analysis of Epidemiological Research and Preventive Technologies, Department of Epidemiology of Chronic Non-Communicable Diseases, National Medical Research Center for Preventive Medicine Svetlana A. Shalnova - MD, PhD, Professor, Head of Department of Epidemiology of Chronic Non-Communicable Diseases, National Medical Research Center for Preventive Medicine

Anna V. Kontsevaya - MD, PhD, Deputy Director for Scientific and Analytical Work, National Medical Research Center for Preventive Medicine

Alexander D. Deev - PhD (in Physics and Mathematics), Head of Laboratory of Biostatistics, National Medical Research Center for Preventive Medicine

Anna V. Kapustina - MD, PhD, Senior Researcher, Department of Epidemiology of Chronic Non-Communicable Diseases, National Medical Research Center for Preventive Medicine

Yulia A. Balanova - MD, PhD, Leading Researcher, Department of Epidemiology of Chronic Non-Communicable Diseases, National Medical Research Center for Preventive Medicine
Конфликт интересов. Все авторы заявляют об отсутствии потенциального конфликта интересов, требующего раскрытия в данной статье.

Disclosures. All authors have not disclosed potential conflicts of interest regarding the content of this paper.

11. Mancia G., Fagard R., Narkiewicz K. et al. 2013 ESH/ESC Guidelines for the management of arterial hypertension. Journal of Hypertension. 2013;31(7):1281-357. doi: 10.3109/08037051.2013.812549.

12. Shkolnikova M., Shalnova S., Shkolnikov V.M. et al. Biological mechanisms of disease and death in Moscow: rationale and design of the survey on Stress Aging and Health in Russia (SAHR). BMC Public Health. 2009;9(1):293. doi: 10.1186/1471-2458-9-293.

13. Valero-Elizondo J., Salami J.A., Ogunmoroti O. Favorable Cardiovascular Risk Profile Is Associated with Lower Healthcare Costs and Resource Utilization: The 2012 Medical Expenditure Panel Survey. Circulation: Cardiovascular Quality and Outcomes. 2016;9(2):143-53. doi: 10.1161/CIRCOUTCOMES.115.002616.

14. Natarajan S., Nietert P.J. Hypertension, diabetes, hypercholesterolemia, and their combinations increased health care utilization and decreased health status. Journal of Clinical Epidemiology. 2004;57(9):954-61. doi: 10.1016/j.jclinepi.2004.01.011.

15. Vals K., Kiivet R.A., Leinsalu M. Alcohol consumption, smoking and overweight as a burden for health care services utilization: a cross-sectional study in Estonia. BMC Public Health. 2013;13(1):772. doi: 10.1186/1471-2458-13-772.

16. Shalnova S. A., Deev A. D., Balanova Yu. A. Twenty years trends of obesity and arterial hypertension and their association in Russia. Cardiovascular Therapy and Prevention 2017;16(4):4-10. (In Russ.) [Шальнова С.А., Деев А.Д., Баланова Ю.А. и др. Двадцатилетние тренды ожирения и артериальной гипертонии и их ассоциации в России. Кардиоваскулярная Терапия и Профилактика 2017; 16 (4): 4-10]. doi: 10.15829/1728-8800-2017-4-4-10.

17. Musich S., MacLeod S., Bhattarai G.R. et al. The impact of obesity on health care utilization and expenditures in a medicare supplement population. Gerontology \& geriatric medicine 2016; 2:1-9. doi: $10.1177 / 2333721415622004$.

18. Schlichthorst M., Sanci L.A., Pirkis J. et al. Why do men go to the doctor? Socio-demographic and lifestyle factors associated with healthcare utilisation among a cohort of Australian men. BMC Public Health. 2016;16(S3):1028. doi: 10.1186/s12889-016-3706-5

19. Nago N., Ishikawa S., Goto T., Kayaba K. Low Cholesterol is Associated With Mortality From Stroke, Heart Disease, and Cancer: The Jichi Medical School Cohort Study. Journal of Epidemiology. 2011;21(1):67-74. doi: 10.2188/jea.JE20100065.

20. Bae J.M., Yang Y.J., Li Z.M., Ahn .- - L. Low Cholesterol is Associated with Mortality from Cardiovascular Diseases: A Dynamic Cohort Study in Korean Adults. Journal of Korean Medical Science. 2012;27(1):58. doi: 10.3346/jkms.2012.27.1.58

\section{Сведения об авторах: \\ Суворова Евгения Игоревна - М.н.С., лаборатория} экономического анализа эпидемиологических исследований и профилактических технологий, отдел эпидемиологии хронических неинфекционных заболеваний, НМИЦ ПМ Шальнова Светлана Анатольевна - д.м.Н., профессор, руководитель отдела эпидемиологии хронических неинфекционных заболеваний, НМИЦ ПМ

Концевая Анна Васильевна - Д.м.н., зам. директора по научной и аналитической работе, НМИЦ ПМ

Деев Александр Дмитриевич - к.ф. -м.н., руководитель лаборатории медицинской биостатистики, НМИЦ ПМ Капустина Анна Владимировна - К.М.Н., С.Н.С, отдел эпидемиологии хронических неинфекционных заболеваний, НМИЦПМ

Баланова Юлия Андреевна - К.М.Н., В.Н.С., лаборатория экономического анализа эпидемиологических исследований и профилактических технологий, отдел эпидемиологии хронических неинфекционных заболеваний, НМИЦ ПМ 